\title{
Enseñanza de semiconductores en tiempos de pandemia: experimentando una secuencia didáctica interactiva
}

Semiconductor teaching in times of pandemic: experiencing an interactive didactic sequence

DOI: $\underline{\text { https://doi.org/10.33262/rmc.v6i1.922 }}$

\author{
María do Socorro Ferreira Ramos ${ }^{1}$ \\ Universidad Estatal de Rio Grande do Norte, Brasil \\ (iD) https://orcid.org/0000-0001-6943-6732. \\ mariasframos@gmail.com \\ Octavio Paulino ${ }^{2}$ \\ Universidad Federal Rural del Semiárido, Brasil \\ (iD) https://orcid.org/0000-0001-5237-3392. \\ otavio.lavor@ufersa.edu.br
}

\section{RESUMEN}

En tiempos de aislamiento social debido a la pandemia, la enseñanza y el aprendizaje han sido un desafío aún mayor y la educación remota ha sido una posibilidad utilizada por varias instituciones, sin embargo, la preparación para esta modalidad puede ser cuestionada. En este sentido, las estrategias deben ser pensadas y en este trabajo, se experimenta la secuencia didáctica interactiva en las clases de semiconductores. Los estudiantes informaron sus conocimientos previos y, a través de tecnologías sincrónicas, los grupos mejoraron sus respuestas hasta que tuvieron un concepto formal de semiconductor. Los resultados muestran una apropiación apropiada del conocimiento y los comentarios y percepciones muestran que la secuencia didáctica interactiva se puede aplicar en la educación remota para superar los desafíos de la enseñanza y el aprendizaje en tiempos de aislamiento social.

PALABRAS CLAVE: Aislamiento social; Interacción; Enseñanza remota.

\begin{abstract}
In times of social isolation due to the pandemic, teaching and learning has been an even greater challenge and remote education has been a possibility used by several institutions, however the preparation for this modality can be questioned. In this sense, strategies must be thought and, in this work, the interactive didactic sequence in classes of semiconductor is experienced. The students informed their previous knowledge and through synchronous technologies, the groups improved their answers until they had a formal concept of semiconductor. The results show an appropriate appropriation of knowledge, and the comments and perceptions show that interactive didactic sequence can be applied in
\end{abstract}


remote education to overcome the challenges of teaching and learning in times of social isolation.

KEYWORDS: Social isolation; Interaction; Remote teaching.

\section{INTRODUCCIÓN}

En los últimos meses, la pandemia de COVID-19 sorprendió a la sociedad y en la búsqueda del control del virus, varias instituciones fueron cerradas y el público se encontraba en aislamiento social. Según Alves (2020), este aislamiento adoptado por todos los países para mantener a la población en el hogar pretende que la economía de los países se refleje en la interrupción de diferentes servicios y actividades, entre ellos el proceso de enseñanza-aprendizaje.

En un intento por continuar el proceso de enseñanza-aprendizaje, incluso en tiempos de pandemia, se han estudiado y verificado estrategias en todo el mundo. Alves (2020) señala que los efectos de COVID-19 en los sistemas escolares dieron como resultado medidas que van desde la suspensión de clases sin interacción por plataformas virtuales, hasta la realización de dichas clases remotas.

Bao (2020) busca identificar los principios de la práctica de alto impacto en la educación superior en línea y proporciona un estudio de caso que se reproducirá en situaciones similares. El autor presenta seis estrategias para mejorar la concentración y el compromiso de los estudiantes que se enumeran a continuación: hacer planes de preparación para emergencias ante problemas inesperados; dividir el contenido en unidades más pequeñas para ayudar a los estudiantes a concentrarse; enfatizar el uso de la voz en la enseñanza; trabajar con asistentes de enseñanza y obtener soporte en línea; fortalecer la capacidad de aprendizaje activo de los alumnos fuera del aula; y combine el aprendizaje en línea y el aprendizaje fuera de línea de manera efectiva.

En este contexto, la educación remota ha sido una posibilidad para que las instituciones continúen los currículos escolares. Por lo tanto, las plataformas digitales han estado mediando prácticas pedagógicas síncronas y asíncronas, manteniendo las aulas sin interacción cara a cara.

Para Nakashima y Lobo (2020), la situación de la pandemia causada por COVID-19 reforzó que la educación debe cambiar y las transformaciones requerirán la unidad y la colaboración del público involucrado, en el que surge la necesidad de dinámicas educativas basadas en nuevas formas de enseñanza.

Además, Diesel et al. (2017) refuerzan que cualquier acción propuesta para enseñar debe ser pensada desde la perspectiva de los participantes para que la planificación y organización de las situaciones de aprendizaje se centren en las actividades de los estudiantes.

En este sentido, la Secuencia Didáctica Interactiva (SDI) puede verse como una herramienta metodológica que tiende a crear interacción en pequeños grupos en función de un tema a estudiar. Esta metodología se ha utilizado en diferentes escenarios y uno puede preguntarse cómo sería su aplicación de forma remota. Entonces, este trabajo busca experimentar con SDI en una clase de materiales eléctricos y magnéticos, específicamente 
en el contenido de semiconductores que son materiales de conductividad eléctrica intermedia entre conductores y aisladores.

\section{Secuencia didáctica interactiva}

SDI es otra opción para que el maestro reflexione y aplique en la enseñanza y la investigación, con el objetivo de una participación del estudiante. Esta propuesta fue creada por Oliveira (2013) y en sus palabras "es una nueva propuesta didácticometodológica para ser utilizada en el contexto del aula, con el objetivo de facilitar el proceso de enseñanza-aprendizaje". Según el autor, SDI es un desarrollo de la metodología interactiva que tenemos:

La metodología interactiva es un proceso hermenéutico-dialéctico que facilita la comprensión e interpretación del discurso y los testimonios de los actores sociales, en nuestro caso, estudiantes, en su contexto y análisis de conceptos en textos, libros y documentos, hacia una visión sistémica del tema en estudiar (Oliveira, 2008, p. 124).

La SDI proporciona una secuencia de actividades en las que los participantes actúan de forma interactiva y, según Oliveira (2013), la propuesta tiene en cuenta la construcción y reconstrucción de conceptos de diferentes temas y niveles de educación. El autor define SDI como:

La secuencia didáctica interactiva es una propuesta didáctico-metodológica que desarrolla una serie de actividades, comenzando con la aplicación del círculo hermenéutico-dialéctico para identificar conceptos / definiciones, que subsidian los componentes curriculares (temas), y que están asociados interactivamente con la (s) teoría (s) de aprendizaje y / o propuestas y metodologías pedagógicas, con el objetivo de construir nuevos conocimientos y conocimientos (Oliveira, 2013, p. 43).

Al aplicar un SDI, la clase se divide en grupos y de acuerdo con Oliveira (2011), cada alumno del grupo informa qué entiende por el tema a estudiar. Luego, se realiza una síntesis de los conceptos que fueron dados por cada participante para formar una definición única en cada grupo. En la siguiente etapa, cada equipo elige un líder, de modo que se forma un nuevo grupo con los líderes y hay una nueva síntesis. A partir de estos pasos, el profesor discute con el aula las dinámicas realizadas para la construcción de conceptos y cierra la dinámica a través de la transmisión y sistematización de contenidos teóricos. La figura 1 ilustra un ejemplo. 


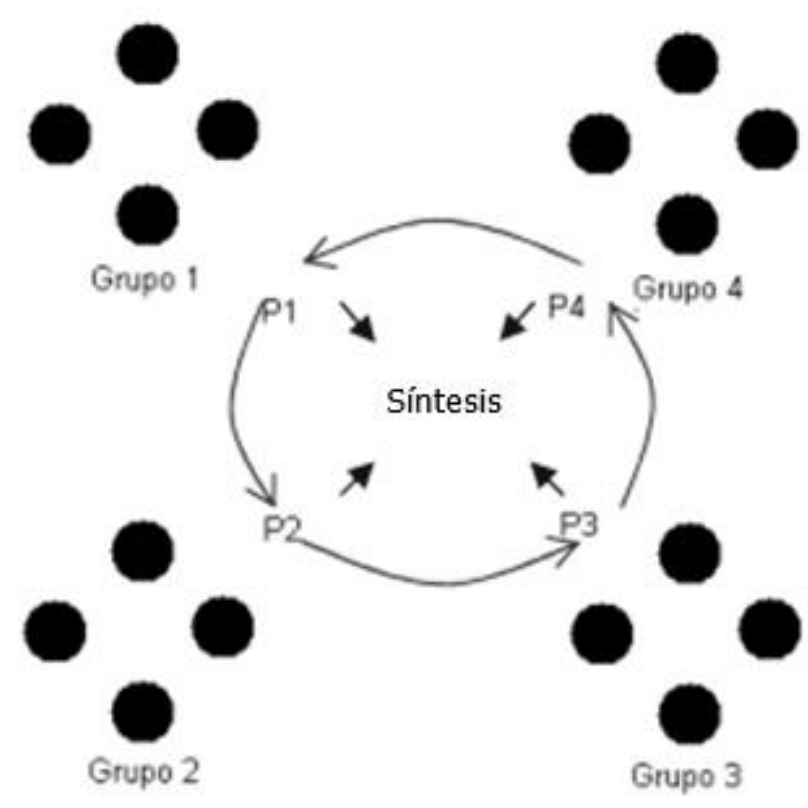

Figura 1. Ejemplo de SDI

Fuente: Oliveira, 2010, p. 9

En el ejemplo anterior, la clase tiene dieciséis estudiantes que se dividen en cuatro grupos con cuatro estudiantes cada uno. Se forma un nuevo grupo de cuatro con los representantes donde se realiza la síntesis para tener una única definición.

Como ejemplo de la aplicación de SDI, podemos mencionar a Silva y Oliveira (2009) que trabajan con el concepto de función similar en la formación del profesorado y concluir que era posible minimizar la subjetividad del profesional en el aula, ya que estaba acompañado por evolución del pensamiento que facilita la síntesis final.

Silva et al. (2013), propusieron una SDI para enseñar el concepto de densidad y se dieron cuenta de que el uso de SDI generaba un aprendizaje significativo y estimulaba la curiosidad y participación de los estudiantes. Moul y Silva (2017) proponen una SDI en la enseñanza de la botánica y se dan cuenta de que muchas concepciones de estudiantes se reafirman después de la secuencia didáctica, mientras que aparecen nuevos conceptos en la formulación de argumentos.

\section{METODOLOGÍA}

Esta investigación se llevó a cabo en una clase de materiales eléctricos y magnéticos con nueve estudiantes en una universidad ubicada en la región semiárida en el curso de licenciatura en ciencia y tecnología. Debido al período de aislamiento social causado por la pandemia, dichos estudiantes fueron inscritos en un semestre complementario ofrecido en modo remoto.

Se intentó un SDI para dinamizar los momentos, demostrando la interacción entre los participantes, incluso de forma remota. De esta manera, en un análisis previo, cada alumno puede exponer sus conocimientos previos sobre semiconductores. La clase se dividió en tres grupos de tres estudiantes, en los que cada grupo se reúne para, a partir de las tres respuestas anteriores, obtener una respuesta única que es llevada por un líder al grupo de síntesis y al final tener una respuesta única que se debate y está justificado por 
el profesor. La figura 2 ilustra la división de la clase en grupos y la formación del nuevo grupo con los líderes.

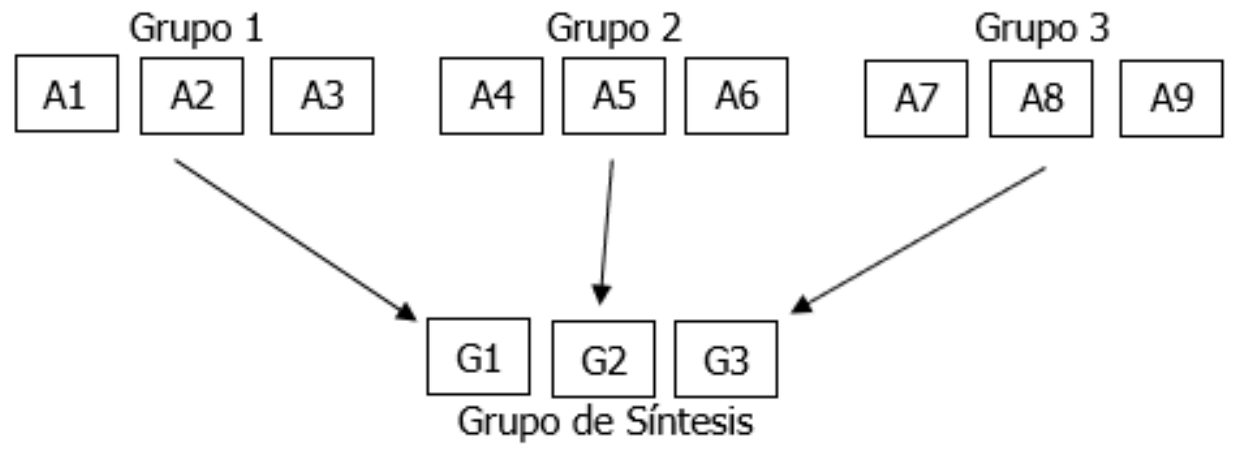

Figura 2. Grupos en SDI

Los grupos 1, 2 y 3 se dividieron por lote y los estudiantes se numeraron del 1 al 9 para que el maestro controle las respuestas y la construcción del conocimiento. Cada grupo podría reunirse de forma remota en la plataforma que quisieran y elegir uno de ellos para componer el grupo de síntesis.

Después de la reunión del grupo de síntesis, el profesor confirmó las respuestas recopiladas, y agregó otra información sobre las características y el dopaje de los semiconductores. Se realizó una evaluación de aprendizaje en un formato de diálogo a través de videollamadas. La siguiente sección presenta más detalles del experimento.

\section{RESULTADOS}

Al principio, los estudiantes emitieron sus conocimientos previos sobre semiconductores. A la continuación puede ver los conocimientos previos de los estudiantes de A1 a A9.

A1: Son materiales con una resistencia y conductividad muy cercanas.

A2: Entiendo que los materiales semiconductores (como su nombre lo indica), son materiales que se encuentran en una "zona" entre materiales conductores y no conductores. Tener características tanto de un grupo como de otro.

A3: Una clasificación de materiales eléctricos que tienen baja conductividad eléctrica, pero que no son aislantes. Ampliamente utilizado en la industria electrónica. Los más conocidos y utilizados son el silicio y el germanio.

A4: Los semiconductores, en mi opinión, es una línea entre conductores y no conductores, donde no tienen la capacidad conductiva fuerte como conductores, pero tampoco tienen una capacidad conductora débil como los no conductores.

A5: Creo que es un material (no metálico) que tiene una conductividad más baja que los conductores, que es capaz de conducir y detener la electricidad, por lo que es posible controlar este flujo de corriente a través del propio material.

A6: Entiendo que los semiconductores son de baja conductividad eléctrica y que es más fácil cambiar su condición más fácilmente.

A7: Los semiconductores son metales con conductividad eléctrica intermedia: en orden creciente de conductividad sería: aislantes <semiconductores <conductores. Ejemplos son: silicio y germanio. 
A8: En la misma palabra cuando lo mencionamos, tenemos la idea de que "semi" es algo que es parcial, es decir, no es completamente conductivo ni totalmente aislante. Conductores es algo que transmite y conduce energía eléctrica a través de su cuerpo. En términos generales, es un objeto que es conductor, pero presenta un medio resistivo que no proporciona una conducción totalmente integrada en comparación con un conductor. Esta palabra también está asociada con la tabla periódica donde tenemos una ubicación específica para algunos de estos materiales.

A9: Los semiconductores son materiales que permiten el paso de la corriente eléctrica, sin embargo, este paso es muy pequeño en comparación con los materiales conductores.

Se puede ver que, los estudiantes ya tienen un conocimiento sobre semiconductores, señalando que es un material entre el conductor y el aislante, que incluye proporcionar ejemplos. Los tres grupos se reunieron a través de una llamada de Whatsapp para obtener una respuesta. A la continuación se muestra la respuesta de los tres grupos.

G1: Los semiconductores son materiales que tienen baja conductividad eléctrica, pero no son aislantes, por lo tanto, están en una "zona" entre materiales conductores y no conductores (aislantes). Son elementos que tienen 4 electrones en la capa de valencia. El más conocido es el silicio, ampliamente utilizado en la industria electrónica.

G2: Los semiconductores son un material no metálico que es capaz de conducir y detener la electricidad de manera que pueda controlar el flujo de corriente en el material. Su conductividad no es tan alta como la de los conductores.

G3: La palabra semiconductores proviene de conductores, que son materiales que permiten el paso de corriente eléctrica fácilmente en su cuerpo, más el prefijo semi, que indica algo parcial. Por lo tanto, podemos conceptualizar los semiconductores como materiales de conductividad eléctrica intermedia. En orden ascendente de conductividad sería: aislantes <semiconductores <conductores. Ejemplos de semiconductores son silicio y germanio.

El grupo 1, que incluía a los estudiantes A1, A2 y A3, formuló una respuesta que contempla el carácter semiconductor como una característica entre el aislante y el conductor. Agregaron la información de cuatro electrones en la capa de valencia y citaron al silicio como el material semiconductor utilizado en la industria. Es posible que el estudiante A1 estuviera convencido de su respuesta en relación con tener una resistencia y conductividad cercanas.

El grupo 2 señala al semiconductor como un material con menor conductividad que los conductores y que puede usarse para el control de corriente. Los estudiantes A4 y A6 renunciaron a sus respuestas para aceptar la respuesta de A5.

El grupo 3 concluyó que el prefijo semi en la palabra semiconductor indica un material de conductividad intermedia entre aisladores y conductores, presentando silicio y germanio como ejemplos.

Cada grupo nombró un líder y estos líderes formaron el grupo de síntesis para llegar a una sola respuesta con respecto al conocimiento previo de la clase. Después de reunirse por llamada de Whatsapp, llegaron a la información a continuación.

La palabra semiconductores proviene de conductores, que son materiales que permiten el paso de corriente eléctrica fácilmente en su cuerpo, más el prefijo semi, que indica algo parcial. Por lo tanto, podemos conceptualizar los semiconductores como materiales con 
conductividad eléctrica intermedia porque son un material no metálico que es capaz de conducir y difundir electricidad porque su conductividad no es tan alta como la de los conductores. En orden creciente de conductividad tenemos: aisladores <semiconductores <conductores. Tenemos como ejemplos: silicio y germanio, entre estos lo silicio se usa más en la industria electrónica.

La respuesta final se realizó reuniendo la información que cada líder tomó de su grupo. Aquí, se puede ver que la información más reciente presenta una definición más formal con ejemplos y aplicaciones, destacando el carácter interactivo y facilitador del aprendizaje. Un hecho que corrobora con Moul y Silva (2017) al observar que, a lo largo de las respuestas, hay una acumulación de las proposiciones iniciales, que se reflejan en la construcción del grupo.

En vista del conocimiento presentado por la clase, en una reunión a través de la plataforma Google Meet, el profesor presenta la definición de semiconductor haciendo una relación con la información dada por la clase. en este punto, se destaca que solo la información discutida no es suficiente para el profesional de la ingeniería y que las características deben ser detalladas, así como explicar las bandas de energía y las técnicas de dopaje, dada la variedad de aplicaciones, especialmente en electrónica, como ya lo mencionaron los alumnos.

La reunión tuvo una discusión sobre los contenidos con la participación de los estudiantes, ya que se alentó al público a interactuar cuando se exponga a situaciones en las que son seres activos en su aprendizaje. Para Diesel et al. (2017), este movimiento de interacción lleva al alumno a reflexionar sobre el tema y emitir una opinión.

La evaluación se realizó de forma dialógica a través de una videollamada de Whatsapp para cada alumno. En este formato, el alumno es evaluado con respecto a sus preguntas y respuestas a las preguntas. Las calificaciones fueron satisfactorias, ya que cada alumno mostró una buena apropiación del conocimiento de semiconductores, pudiendo preguntar y responder sobre el tema, en el que se destaca que las respuestas en el diálogo fueron más formales y con mayor conocimiento en comparación con las respuestas iniciales.

Los estudiantes señalan que SDI fue relevante porque generó la motivación para el estudio grupal y la oportunidad de usar tecnologías y redes sociales como Whatsapp para fines de aprendizaje, especialmente en momentos en que la distancia social es necesaria. También solicitan que se realicen actividades de esta naturaleza en otros contenidos y otras disciplinas.

Estos hechos llegan a contribuir con Silva et al. (2013), quienes señalan que los estudiantes hablaron sobre cuánto era placentero trabajar en grupos en el concepto de densidad, porque de esa manera pueden interactuar más y construir conocimiento.

\section{CONCLUSIONES}

En esta experimentación, se aplicó un SDI a una clase de materiales eléctricos y magnéticos, específicamente en el contenido de semiconductores, en el que los estudiantes informaron sus conocimientos previos y se dividieron en grupos para interactuar y mejorar sus respuestas. En el grupo de síntesis, se llegó a la respuesta formal 
al concepto de semiconductores, presentando la diferencia entre aisladores y conductores, ejemplos y aplicaciones.

Los conceptos se basaron y luego, en una reunión, se discutieron las propiedades y características que clasifican los semiconductores como materiales aplicables, especialmente en electrónica. Los resultados apuntan a un estímulo para la interacción y la motivación para el aprendizaje. La evaluación dialogada presentó preguntas y respuestas que corresponden a una apropiación apropiada del conocimiento de los materiales semiconductores.

Todos los momentos tuvieron lugar de forma remota a través de Whatsapp y Google Meet, dado el aislamiento social en tiempos de pandemia. Los comentarios y las percepciones indican que SDI es una metodología aplicable de forma interactiva, incluso en salas virtuales. Por lo tanto, se espera que los hechos presentados aquí motiven la reflexión y la mejora de la enseñanza y el aprendizaje de forma remota y que generen entusiasmo para enseñar y aprender en diferentes momentos y con diferentes contenidos.

\section{REFERENCIAS BIBLIOGRÁFICAS}

ALVES, L. (2020). Educação Remota: entre a ilusão e a realidade. Interfaces Científicas, v.8, n. 3, p. $348 \quad-\quad 365 . \quad$ Disponible en: <https://periodicos.set.edu.br/index.php/educacao/article/view/9251>. Acceso em 10 jul. 2020.

BAO, W. (2020). COVID-19 and onlineteaching in higher education: a case study of Peking University. Hum Behav \& Emerg Tech, v. 2, p. 113-115. Disponible en: $<$ https://onlinelibrary.wiley.com/doi/epdf/10.1002/hbe2.191>. Acceso en: 11 jul. 2020.

DIESEL, A.; BALDEZ, A. L. S.; MARTINS, S. B. (2017). Os principios das metodologías ativas de ensino: una abordagem teórica. Revista Thema, v. 14, n. 1, p. 268-288. Disponible en: <http://periodicos.ifsul.edu.br/index.php/thema/article/view/404/295>. Acceso en: 12 jul. 2020.

MOUL, R. A. T. M.; SILVA, F. C. L. A (2017). Construção de Conceitos em Botânica a partir de uma Sequência Didática Interativa: proposições para o ensino de ciências. Revista Exitus, v. 7, n. 2, p. 262-282. Disponible en: <http://www.ufopa.edu.br/portaldeperiodicos/index.php/revistaexitus/article/vie w/313/261>. Acceso en: 01 jun. 2020.

NAKASHIMA, R. H. R.; LOBO, M. P. (2020). Virada Curricular: por una pedagogía universitaria relevante, equitativa y solidaria. Disponible en: <https://osf.io/25xzy/download/?format=pdf>. Acceso e 10 jul. 2020.

OLIVEIRA, M. M. (2008). Como fazer pesquisa qualitativa. 2 ed. Petrópolis: Vozes.

OLIVEIRA, M. M. (2010). Sequência Didática Interativa no Ensino De Ciências. In: IV COLÓQUIO INTERNACIONAL EDUCAÇÃO E CONTEMPORANEIDADE, Anais 2010. Laranjeiras: UFS.

OLIVEIRA, M. M. (2011). Círculo hermenêutico-dialético como sequência didática interativa. Revista Interfaces Brasil/Canadá, v. 11, n. 1, p. 235-251. Disponible en: 
$<$ https://periodicos.ufpel.edu.br/ojs2/index.php/interfaces/article/view/7173/499 0>. Acceso en: 01 jun. 2020.

OLIVEIRA, M. M. (2013). Sequência didática interativa no processo de formação de professores. 2 ed. Petrópolis: Vozes.

SILVA, A. P. B.; OLIVEIRA, M. M. (2009). A Sequência Didática Interativa como Proposta para Formação de Professores de Matemática. In: VII ENCONTRO NACIONAL DE PESQUISA EM EDUCAÇÃO EM CIÊNCIAS, Anais do VII Enpec. Florianópolis: UFSC.

SILVA, I, K.; BARBOSA, A. O. S.; OLIVEIRA, M. M. (2013). Abordando o Conceito de Densidade a Partir de uma Sequência Didática Interativa. IN: XIII JORNADA DE ENSINO, PESQUISA E EXTENSÃO DA UFRPE. Anais do XIII Jornada de Ensino, Pesquisa e Extensão da UFRPE. Recife/Garanhuns/Serra Talhada: UFRPE.

\section{PARA CITAR EL ARTÍCULO INDEXADO.}

Lavor, O. P., \& Ramos, M. do S. F. (2021). Enseñanza de semiconductores en tiempos de pandemia: experimentando una secuencia didáctica interactiva. Magazine De Las Ciencias: Revista De Investigación E Innovación, 6(1), 67-75. https://doi.org/10.33262/rmc.v6i1.922 